\title{
A Prevalent Cancer Associated Glycan, Globo H Ceramide, Induces Immunosuppression by Reducing Notch1 Signaling
}

Yi-Chien Tsai ${ }^{1,2}$, Jing-Rong Huang ${ }^{2,3}$, Jing-Yan Cheng ${ }^{1,2}$, Jin-Jin Linn ${ }^{2,4}$, Jung-Tung Hung ${ }^{2}$, Yih-Yiing Wu ${ }^{5}$, Kun-Tu Yeh ${ }^{6 *}$ and Alice L. Yu ${ }^{1,2,7 *}$

${ }^{1}$ Graduate Institute of Life Sciences, National Defense Medical Center, Taipei, Taiwan

${ }^{2}$ Genomic Research Center, Academia Sinica, Taipei, Taiwan

${ }^{3}$ Institute and Department of Microbiology and Immunology, Nation Yang-Ming University, Taipei, Taiwan

${ }^{4}$ Institute of Biochemistry and Biotechnology, college of Medicine, Chung Shan Medical University, Taichung, Taiwan

${ }^{5}$ Department of Pathology, Taipei City Hospital, Taipei, Taiwan

${ }^{6}$ Department of surgical pathology, Changhua Christian Hospital, Changhua, Taiwan

${ }^{7}$ Center of Stem Cell and Translational Cancer Research, Chang Gung Memorial Hospital, Taoyuan, Taiwan

\begin{abstract}
Globo $\mathrm{H}$, a hexasaccharide initially identified as a ceramide-linked glycolipid from human breast cancer cel line MCF-7, is over-expressed on the surface of many common cancers, but its function is unknown. Here we demonstrated the uptake of globo $\mathrm{H}$ ceramide (GHCer) by human peripheral blood mononuclear cells (PBMC) upon co-culture with MCF-7 cells. Significantly, the expression of globo $\mathrm{H}$ on tumor-infiltrating lymphocytes was observed in $61 \%$ of globo $\mathrm{H}$ positive breast cancer tissues. Addition of synthetic GHCer to human PBMC, mouse splenocytes or purified CD4+ T cells inhibited their proliferative response to anti-CD3/CD28 to $60 \pm 1 \%, 50 \pm 7 \%$ and $62 \pm 5 \%$ of control, respectively, and reduced the secretion of IL-2, IFN-y and IL-4. GHCer also significantly suppressed the proliferation of splenocytes or purified CD19+ B cells to $45 \pm 10 \%$ and $26 \pm 3 \%$ of control in response to LPS or LPS + IL4 +CD40 ligand and their IgM production to $12 \pm 5 \%, 8 \pm 3 \%$, and IgG to $34 \pm 9 \%, 35 \pm 5 \%$, respectively, with neglible induction of plasma cells. Ceramide displayed no such inhibitory effects. On the other hand, GHCer failed to raise the number of regulatory T cells, or their expression of FOXP3/CTLA4, nor did it increase apoptosis. Notably, GHCer significantly suppressed the Notch1 signaling during activation of human PBMC and murine T and B cells. Furthermore, GHCer upregulated the expression of $i d 3$ and $i t c h$ by $2.1 \pm 0.2$ and $4.7 \pm 0.4$ folds, respectively, leading to ID3-dependent downregulation of Notch1 and Itch-mediated Notch1 degradation. These results provide the first evidence for GHCer to facilitate the escape of cancer cells from immune surveillance.
\end{abstract}

Keywords: Globo H ceramide; Tumor associated glycolipid antigen; Immunosuppression; Notch

\section{Abbreviations: GHCer: Globo H ceramide}

\section{Introduction}

Globo $\mathrm{H}$, a hexasaccharide (Fuca $1 \rightarrow 2 \mathrm{Gal} \beta 1 \rightarrow 3 \mathrm{GalNAc} \beta 1 \rightarrow 3 \mathrm{Gal} \alpha 1$ $\rightarrow 4 \mathrm{Gal} \beta 1 \rightarrow 4 \mathrm{Glc} \beta 1$ ) was first identified as a ceramide linked glycolipid in human breast cancer cell line MCF-7 [1]. It has been reported to be expressed on a variety of cancers including breast, colon, ovarian, gastric, pancreatic, lung, and prostate cancers [2,3]. It was also found on breast cancer stem cells [4]. Its limited expression in normal tissues made globo $\mathrm{H}$ an ideal target of immunotherapy against tumors [5]. So far, all the commercially available cancer immunotherapeutics are directed against protein/glycoprotein antigens. However, tumor associated GD2 has recently been shown to be effective target for immunotherapy [6]. Globo $\mathrm{H}$ is being hotly pursued as the next nonprotein antigen target for cancer immunotherapy with ongoing multinational clinical trial, but its function remains unknown.

Cancer cells often displayed altered glycosylation pattern on their surface proteins or glycosphingolipids [7]. Several cancer-associated gangliosides had been shown to be involved in cancer cell migration, invasion, angiogenesis and metastasis $[8,9]$. These gangliosides could also inhibit immune cell responses, including antigen processing and presentation, $\mathrm{T}$ cell proliferation, and cytokine production, such as IFN- $\gamma$ and IL-4 [10-15]. Purified gangliosides from cancer cells displayed immunosuppressive activities which aided cancers to escape from host immune surveillance [13,16-18]. The mechanisms of gangliosides-induced immunosuppression were mediated by interfering the interaction of IL-2 with its receptor [19], inducing apoptotic cell death [20] and deviation toward Th2 response [21]. The molecular bases for gangliosides-induced $\mathrm{T}$ cell dysfunction involved NF-kappa B inhibition through degradation of RelA and p50 proteins [22]. In contrast, there has been no study on the function of globo $\mathrm{H}$ to date.

There are multiple mechanisms of cross talk between Notch and NF-kappa B [23].The Notch signaling pathway is an evolutionarily conserved cell signaling system in most organisms, and it can regulate cell proliferation, differentiation, apoptosis, and survival [24]. Notch signaling is an important regulator of leukocyte differentiation and maturation [25]. It is also involved in TCR-mediated T cells activation, proliferation and B cell differentiation into Ig-secreting plasma cells [26,27]. Upon interaction with its ligand, Notch can directly activate transcription of target genes [28]. The regulation of Notch1 is controlled by the E-protein transcription factor E2A and its natural inhibitor ID3 [29]. It is degraded by ubiquitination though E3 ubiquitin ligase, Itch

*Corresponding authors: Dr. Alice L. Yu, Genomics Research Center, Academia Sinica 128, Academia Road, Sec. 2, Nankang, Taipei 115, Taiwan, Tel: +886-22789-9930; Fax: +886-2-2789-9931; E-mail: ayu@gate.sinica.edu.tw

Kun-Tu Yeh, Department of surgical pathology, Changhua Christian Hospital, 135 Nansiao St., Changhua, 500 Taiwan, Tel: +886-4-723-8595; Fax: +886-4-7232942; E-mail: 10159@cch.org.tw

Received May 29, 2013; Accepted June 20, 2013; Published June 22, 2013

Citation: Tsai YC, Huang JR, Cheng JY, Lin JJ, Hung JT, et al. (2013) A Prevalent Cancer Associated Glycan, Globo H Ceramide, Induces Immunosuppression by Reducing Notch1 Signaling. J Cancer Sci Ther 5: 264-270. doi:10.4172/19485956.1000215

Copyright: () 2013 Tsai YC, et al. This is an open-access article distributed under the terms of the Creative Commons Attribution License, which permits unrestricted use, distribution, and reproduction in any medium, provided the original author and source are credited. 
Citation: Tsai YC, Huang JR, Cheng JY, Lin JJ, Hung JT, et al. (2013) A Prevalent Cancer Associated Glycan, Globo H Ceramide, Induces Immunosuppression by Reducing Notch1 Signaling. J Cancer Sci Ther 5: 264-270. doi:10.4172/1948-5956.1000215

[30]. Adler et al. reported that Notch signaling enhanced naïve T cell proliferation by modulating the expression of CD25 [31]. But until now, there is no report on the relationship between glycoshingolipids induced immunosuppresion and Notch signaling.

In this study, we first demonstrated the uptake of GHCer by immune cells in vitro and by tumor infiltrating-lymphocytes in breast cancer in vivo. Addition of GHCer to immune cells could inhibit their proliferation and cytokine or immunoglobulin secretion. We also elucidated the molecular mechanism of GHCer induced immunosuppression which involved inhibition of Notch1 signaling through the induction of ID3 and Egr2/3 accompanied itch expression. The key immunosuppressive effects and downregulation of Notch1 by GHCer were demonstrated in both human and murine immune cells, with further detailed analysis in murine system, given the readily accessible immune cells from inbred strain of mice. Our findings illustrated for the first time the role of GHCer in the escape of cancer cells from immune surveillance.

\section{Material and Methods}

\section{Materials}

Female BALB/c mice (8-12 weeks old) were obtained from National Laboratory Animal Center (Taipei, Taiwan) and housed under specific pathogen free condition. All animal studies were approved by the Institutional Animal Care and Use Committee of the Academia Sinica. Normal human blood was obtained from Taipei Blood Center with the approval of the Human Subject Research Ethics committee of both Academia Sinica and the Taiwan Blood Services Foundation. Tissue sections of human breast cancer were obtained from Chunghua Christian Hospital (Chunghua, Taiwan) and were fully encoded to protect patient confidentiality. This study was approved by the Institutional Review Board of Human Subjects Research Ethics Committees of Academia Sinica (Taipei, Taiwan) and Chunghua Christian Hospital. The MCF-7 cells were obtained from ATCC and maintained in MEM medium supplemented with $10 \%$ heat-inactivated FCS, $100 \mathrm{ug} / \mathrm{ml}$ streptomycin, and $100 \mathrm{U} / \mathrm{ml}$ penicillin (Invitrogen, Frederick, MD)

\section{Reagents and Flow cytometry}

GHCer with fatty acid tail 16:0 was obtained from OBI Pharma Inc. and dissolved with PBS at a concentration of $2 \mathrm{mg} / \mathrm{ml}$. LPS was purchased from Sigma-Aldrich. IL-4 and CD40L were purchased from PeproTech. Antibodies against CD3-LEAF (145-2C11 and OKT3), CD28-LEAF (37.51 and CD28.2), CD4-PE (RM4-5 and OKT4), CD19PE (6D5), CD25-FITC (3C7), CTLA4-PE (UC10-4B9), FOX-P3-PE (150D) and Notch1-PE (mN1A) were purchased from Biolegend. Monoclonal antibody against globo $\mathrm{H}$ was prepared from VK9 hybridoma provided by Dr. Ragupathi (52). Single cell suspensions of lymphoid cells were stained with fluorescence labeled mass following the manufacturer's instructions. Flow cytometry was performed with FACSCanto (BD, Franklin Lakes, NJ) and the data were analyzed by FCS Expresss V3 software (De Novo Software, Canada).

\section{Isolation of T and B cells from mouse splenocytes}

$\mathrm{CD} 4+\mathrm{T}$ cells were purified by anti-CD4 magnetic microbead following the manufacturer's instructions (Miltenyi Biotec, Gladbach, Germany). The CD19+ B cells were positive selected by anti-CD19-PE $\mathrm{Ab}$ followed by anti-PE magnetic microbead. The purity of isolated cells was greater than $95 \%$ by FACS analysis.

\section{Cell proliferation and cytokine production assays}

Mouse splenocytes, purified CD4+ T cells and human PBMCs (5,
2 and $2 \times 10^{5} /$ well, respectively) were stimulated with/without anti$\mathrm{CD} 3 / 28 \mathrm{mAbs}$ ( 1 and $3 \mu \mathrm{g} / \mathrm{ml}$ respectively) coated wells at $37^{\circ} \mathrm{C}$ for 3 days. Purified CD19+ B cells were activated by LPS $(2 \mu \mathrm{g} / \mathrm{ml})$ with/ without IL-4 $(10 \mathrm{ng} / \mathrm{ml})+$ CD40L $(1 \mathrm{ng} / \mathrm{ml}) .\left[{ }^{3} \mathrm{H}\right]$ thymidine $(1 \mu \mathrm{Ci} /$ well) was added to the cells at the last 16 hrs before harvest. The $\left[{ }^{3} \mathrm{H}\right]$ thymidine incorporation was determined by TopCount NXT gamma counter (Packard, Meriden, CT). Cell culture supernatants were determined for IL-2, IL-4, IFN- $\gamma$, IL-10 and TGF- $\beta$ by DuoSet ELISA Development System according to the manufacturer's procedures (R\&D System, USA).

\section{Apoptosis assay}

Mouse splenocytes $\left(5 \times 10^{5}\right.$ cells/well) were stimulated with anti$\mathrm{CD} 3 / 28$ antibody-coated wells and incubated at $37^{\circ} \mathrm{C}$ for 24,48 and 72 $\mathrm{h}$ in 96 well flat bottom plates. Cells were stained with annexin V-FITC and propidium iodide provided by the Apoptosis Detection kit (BD Biosciences). Data was performed by flow cytometry analysis.

\section{Quantitative real-time PCR}

Total RNA was extracted from purified mouse CD4+ T cells with TRIzol reagent (Invitrogen Life Technologies), and $1 \mu \mathrm{g}$ of RNA was reversely transcribed into cDNA using Omiscript RT (Qiagen,Hilder, Germany). cDNA (20 ng) mixed with 1X SYBR Green Master Mix (Applied Biosystems) was used to perform real-time quantitative PCR on an Applied Biosystems PRISM 7300-HT. The values of cycle threshold were used to calculate the fold differences of gene expression relative to the expression of the housekeeping gene, $\beta$-actin. The primers used in this assay were listed as following: id3; forward, 5'- TCCTGGCACCTCCCGAAC-3'; reverse, 5'- TAAGTGAAGAGGGCTGGGTTAAG-3', itch; forward, 5'- GTGTGGAGTCACCAGACCCT-3'; reverse, 5'- GCTTCTACTTGCAGCCCATC-3', egr2; forward, 5' GTGCCAGCTGCTATCCAGAAG -3'; reverse, 5'- GGCTGTGGTTGAAGCTGGAG -3', egr3; forward, 5'- CAACGACATGGGCTCCATTC -3'; reverse, 5'- GGCCTTGATGGTCTCCAGTG -3', $c b l-b$; forward, 5'- CTTAAATGGGAGGCACAGTAGAAT-3'; reverse, 5'CAGTACACTTTATGCTTGGGAGAA-3’.

\section{Immunohistochemical staining of clinical specimens of breast cancer}

For globo $\mathrm{H}$ staining, tissue sections were deparaffinized followed by antigen retrieval by autoclave for $121^{\circ} \mathrm{C}, 5$ mins in $\mathrm{AR}-10$ solution (Biogenex). Slides were incubated with VK9 antibody (1:100 dilutions in antibody dilution buffer, Ventana Medical Systems, Inc.) overnight at $4^{\circ} \mathrm{C}$ followed by polymer-HRP IHC detection system (Biogenex). The slides were counter stained with hematoxylin and mounted. CD3 immunohistochemical staining was performed automatically with Ventanas Benchmark ${ }^{\oplus}$ XT by using the rabbit anti-human CD3 (DAKO Corporation, Carpinteria, CA). Digital images were captured by Aperio ScanScope XT Slide Scanner (Aperio Technologies, Vista, CA, USA) under $20 \times$ magnification.

\section{Statistical analysis}

The statistical significance between each group was analyzed by unpaired two-tailed Student's $t$ test. $\mathrm{P}$ value less than 0.05 was considered significant.

\section{Results}

\section{Uptake of globo $\mathrm{H}$ by lymphocytes in vitro and in vivo}

It has been reported that tumor cells could shed gangliosides into microenvironment to help its growth (32-34). To test whether globo 
$\mathrm{H}$ could be transferred from cancer cells to immune effector cells, we cultured the human PBMC in the presence/absence of breast cancer cell line MCF-7 which expressed high level of globo $\mathrm{H}$ on its surface. Three days after incubation with MCF-7, globo $\mathrm{H}$ was detected on the surface of $\mathrm{PBMC}($ Figure $1 \mathrm{~A}$ ) as well as $\mathrm{CD} 4+$ cells (Figure 1B) while it was undetectable on PBMC cultured without MCF-7 cells. Similarly, globo $\mathrm{H}$ could be detected on BALB/c mouse splenocytes upon incubation with chemically synthesized GHCer for 3 days but not with the ceramide (Figure 1C). Next, we explored the possibility of globo $\mathrm{H}$ uptake by tumor infiltrating-lymphocytes. A total of 98 tumor tissues of breast cancer were examined by Immunohistochemical staining with anti-globo $H$. Figure1D showed representative tissue sections. In the left upper panel, globo $\mathrm{H}$ was easily discernible on the infiltrating lymphocytes surrounding the tumor which was strongly positive for globo $\mathrm{H}$. On the other hand, globo $\mathrm{H}$ was undetectable on the infiltrating lymphocytes in tumors which were slightly positive or negative for globo $\mathrm{H}$, as shown in upper middle and upper right panel, respectively, of Figure 1D. The sections were stained with antiCD3 antibody which revealed that most tumor infiltrating lymphocytes were T cells (Figure 1D, lower panel). All together, 61 of 98 breast cancer samples (62.2\%) showed positive staining of tumors for globo $\mathrm{H}$ and 37 of 98 samples (37.7\%) showed positive staining of both tumors and lymphocytes for globo $\mathrm{H}$ (Figure 1E). These results indicated that globo $\mathrm{H}$ could be shed or transferred from cancer cells to the immune cells in vitro and in vivo.

\section{GHCer inhibited the proliferation of mouse and human immune cells}

To determine whether the incorporation of GHCer could influence the proliferation of immune cells, splenocytes or purified CD4+ T cells from BALB/c mouse were pretreated with GHCer or ceramide for 24 hours before stimulation with plate bound antibodies against mouse CD3 and CD28 for 3 days. As determined by $\left[{ }^{3} \mathrm{H}\right]$-thymidine incorporation, GHCer but not ceramide inhibited anti-CD3/CD28 induced proliferation of splenocytes and CD4+ T cells to $50 \pm 7 \%$

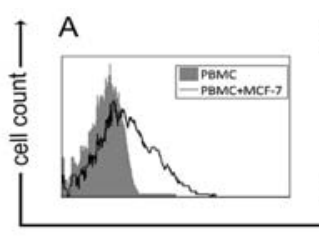

B

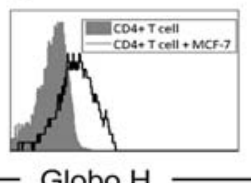

Globo $\mathrm{H}$
C

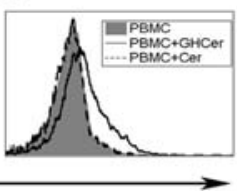

E

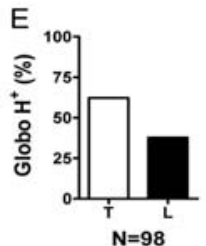

CD3

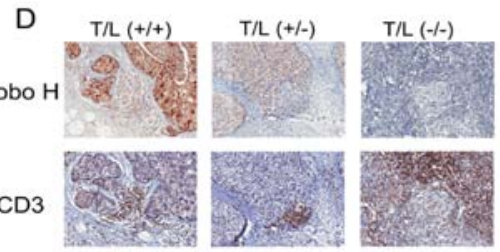

Figure 1: Uptake of globo $\mathrm{H}$ by lymphocytes in vitro and in vivo. Human PBMCs were cultured with/without human breast cancer cell, MCF-7. Three days later, PBMCs were harvested from culture medium and the expression of Globo $\mathrm{H}$ on PBMC (A) and CD4+ T cells (B) was determined by flow cytometry with mAb VK9. Splenocytes from BALB/c mouse were incubated with synthetic Globo$\mathrm{H}$-ceramide or ceramide for 3 days, and stained with mAb VK9 to detect the expression of Globo $\mathrm{H}$ by flow cytometry (C). (D) Tissue sections of breast cancer with Immunohistochemical staining of VK9 or anti-human CD3 antibody. $\mathrm{T}$ : tumor, L: lymphocyte, +: globo $\mathrm{H}$ positive, -: globo $\mathrm{H}$ negative. (E) The percent of globo $\mathrm{H}$ expression in the tumors and infiltrating lymphocytes. $\mathrm{T}$ : the percent of globo $\mathrm{H}$ positive tumors in 98 clinical breast cancer samples. L: the percent of globo $\mathrm{H}$ positive tumors containing globo $\mathrm{H}$ positive lymphocytes in 98 samples.

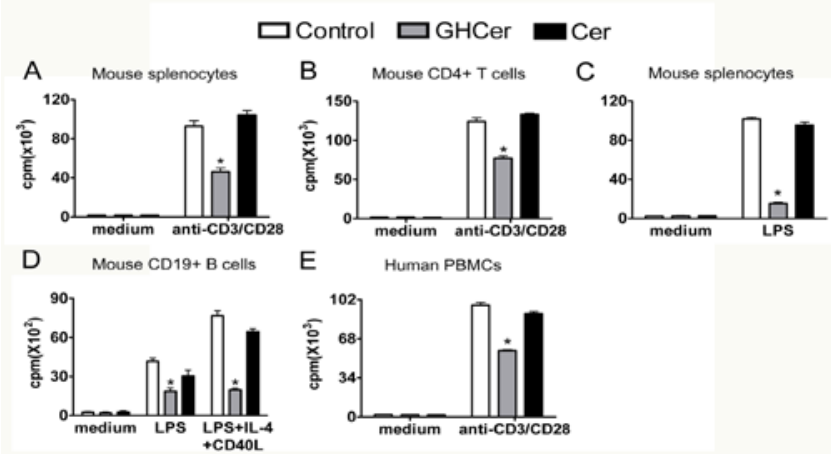

Figure 2: Inhibition of proliferation of immune cell by GHCer. Indicated immune cells were incubated with GHCer, ceramide or medium control for $24 \mathrm{hrs}$ and then activated by anti-mouse CD3 and CD28 mAbs (A and B), LPS (C), LPS in the presence of IL4 and mouse CD40 ligand (D), or anti-human CD3 and CD28 mAbs (E). Cell proliferation was determined by $\left[{ }^{3} \mathrm{H}\right]$-thymidine incorporation assay. The data are presented as mean \pm SD of triplicate. ${ }^{*}, p<0.05$, compared with control.

and $62 \pm 5 \%$ of the control, respectively (Figures $2 \mathrm{~A}$ and $2 \mathrm{~B}$ ). GHcer or ceramide had no effect on proliferation without anti-CD3/CD28 stimulation. To asses whether GHCer also affect B cell proliferation, murine splenocytes or purified CD19+ B cells were stimulated with LPS or LPS+IL-4+CD40L in the presence or absence of GHCer or ceramide for 4 days and cell proliferation was determined by $\left[{ }^{3} \mathrm{H}\right]-$ thymidine incorporation. As shown in Figure 2C, upon stimulation with LPS, GHCer but not ceramide inhibited the proliferation of mouse splenocytes to $15 \pm 2 \%$ of the control. The proliferation of CD19+ B cells stimulated with LPS or LPS+IL-4+CD40L was reduce by GHCer to $45 \pm 10 \%$ and $26 \pm 3 \%$ of control, respectively (Figure 2D). Similar effects of GHCer on human PBMC were observed. As shown in Figure 2E, GHcer but not ceramide-decreased anti-human CD3/ CD28 induced proliferation of human PBMC to $60 \pm 1 \%$ of control. These results suggested that GHCer could inhibit the proliferation of mouse and human immune cells.

\section{Effects of GHCer on the production of cytokines and immunoglobulin by stimulated mouse cells}

To investigate the effects of GHCer on the cytokine production of activated $\mathrm{T}$ cells, murine splenocytes or purified CD4+ T cells were pretreated with GHCer or ceramide for $24 \mathrm{hrs}$ before activation by antiCD3 and anti-CD28 antibodies. Three days later, culture supernatant was collected for determination of IL-2, IFN- $\gamma$ and IL- 4 by ELISA assay. As shown in Figure 3A, the secretion of IL-2 from activated splenocytes or CD4+ T cells was suppressed by GHCer but not ceramide to $19 \pm$ $1 \%$ and $72 \pm 4 \%$ of control, respectively. GHCer also inhibited IFN- $\gamma$ secretion to $40 \pm 3 \%$ and $37 \pm 2 \%$ (Figure $3 \mathrm{~B}$ ) and IL-4 secretion to $12 \pm 2 \%$ and $31 \pm 8 \%$ of control, respectively, (Figure $3 \mathrm{C}$ ). We next examined whether GHCer treatment could affect the secretion of immunoglobulins and the generation of CD138+ plasma cell from LPS-stimulated B cell. Purified mouse CD19+ B cells were treated with GHCer or ceramide before activation by LPS or LPS in the presence of IL-4 and CD40 ligand. Four days later, supernatant was collected for measurement of IgM and IgG by ELISA assay. As shown in Figures $3 \mathrm{D}$ and $3 \mathrm{E}$, after stimulation by LPS or LPS in the presence of IL- 4 and CD40 ligand, the secretion of IgM was decreased by GHCer to $12 \pm$ $5 \%, 8 \pm 3 \%$ of control, and for IgG, to $34 \pm 9 \%, 35 \pm 5 \%$, respectively. The percentage of CD138+ plasma cells was reduced to negligible levels in GHCer treated CD19+ B cells (Figure 3F). Ceramide neither alter 
Citation: Tsai YC, Huang JR, Cheng JY, Lin JJ, Hung JT, et al. (2013) A Prevalent Cancer Associated Glycan, Globo H Ceramide, Induces Immunosuppression by Reducing Notch1 Signaling. J Cancer Sci Ther 5: 264-270. doi:10.4172/1948-5956.1000215

the secretion of cytokines or immunoglobulins, nor the generation of plasma cells. These results showed that GHCer had significant impact on $\mathrm{T}$ or B cell activation by anti-CD3/CD28 or LPS.

\section{GHCer has no effect of on the induction of cell apoptosis or Treg cells}

Regulatory $\mathrm{T}$ cells (Treg) had been shown to exert negative immunoregulatory effects directly through CTLA 4 or indirectly by the secretion of IL-10 and TGF- $\beta$. To determine whether the inhibitory effects of GHCer on T cell activation was mediated by Treg, we analyzed the production of IL-10, TGF- $\beta$ and the population of Treg after antiCD3/CD28 mAbs activation. As shown in Figure 4A and 4B, IL-10 and TGF- $\beta$ production did not change significantly in the GHCer treated cells when compared to the control cells after stimulation for 3 days. Similarly, GHCer treatment did not alter the percentage and absolute number of regulatory $\mathrm{T}$ cells (Figure $4 \mathrm{C}$ and $4 \mathrm{D}$ ) nor their expression of foxp3 and CTLA4 (Figures $4 \mathrm{E}$ and $4 \mathrm{~F}$ ) after activation for 2 days. To explore the possibility of apoptosis induced by GHCer, murine splenocytes were activated by anti-CD3/CD28 mAbs for three days in the presence/absence of GHCer and apoptotic cells were determined by staining with Annexin V and PI. As shown in Figure 4G, the percentage of apoptotic cells (Annexin V+) was lower in GHCer treated cells (46 \pm $1.5 \%$ and $60 \pm 1.1 \%)$ than control cells ( $55 \pm 2.1 \%$ and $67 \pm 1.8 \%)$ at 24 and $48 \mathrm{hrs}$ after stimulation, respectively. These results indicated that the inhibition of cell proliferation by GHCer was neither mediated by expansion of Tregs nor the induction of cell apoptosis.

\section{GHCer decreased the expression of CD25 and Notch1 during cell activation}

CD25, the IL-2 receptor, which was up-regulated upon T cell activation, was crucial for $\mathrm{T}$ cell proliferation. To determine whether GHCer affected CD25 expression, murine splenocytes or purified CD4+ T cells were incubated with GHCer or ceramide for 24 hrs before activation by anti-mouse CD3/CD28 mAbs. Two days later, cells were collected and the expression of CD25 on CD4+ T cells was analyzed by flow cytometry. As shown in Figures 5A and 5B, expression of CD25 on CD4+ T cells was decreased by GHCer. Since Notch1 signaling has been

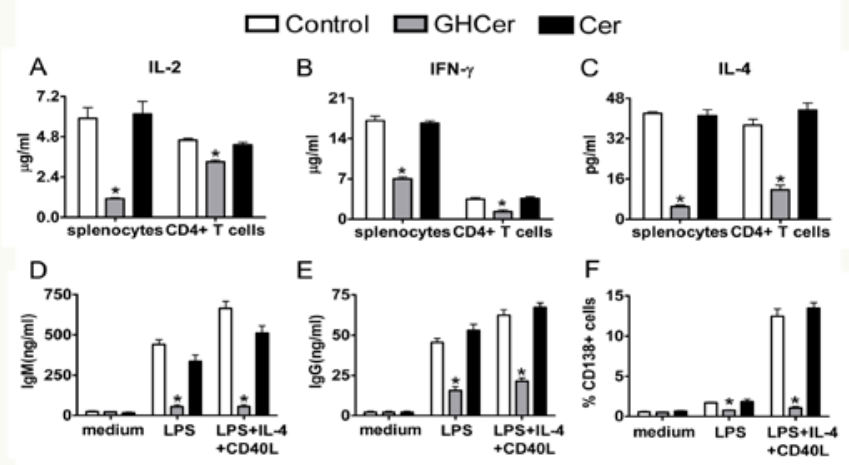

Figure 3: Effects of GHCer on the production of cytokine and immunoglobulin Mouse splenocytes or purified CD4+ T cells were incubated with $\mathrm{GHCer}$, ceramide or medium control for $24 \mathrm{hrs}$, and then activated by anti-mouse CD3 and CD28 mAbs for 3 day. Supernatants were collected for determination of IL-2 (A), IFN-y (B) and IL-4 (C) by ELISA assay. Mouse splenocytes or purified CD19+ B cells were incubated with GHCer or ceramide for $24 \mathrm{hrs}$ and activated by LPS or LPS combined with IL-4 and mouse CD40 ligand for 4 days. Supernatants were collected to determine the production of $\operatorname{lgM}(\mathrm{D})$ and $\lg G(E)$ by ELISA assay and cells were harvested to examine the population of CD138+ plasma cells $(F)$ by flow cytometry. The data are presented as mean $\pm S D$ of triplicate. ${ }^{*}, p<0.05$, compared with control.

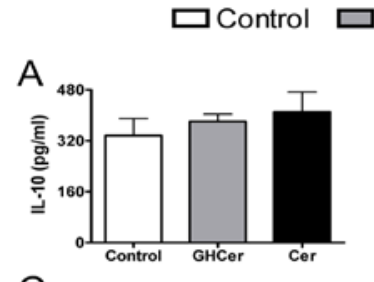

C

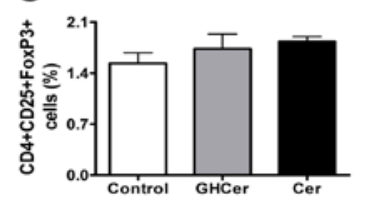

D
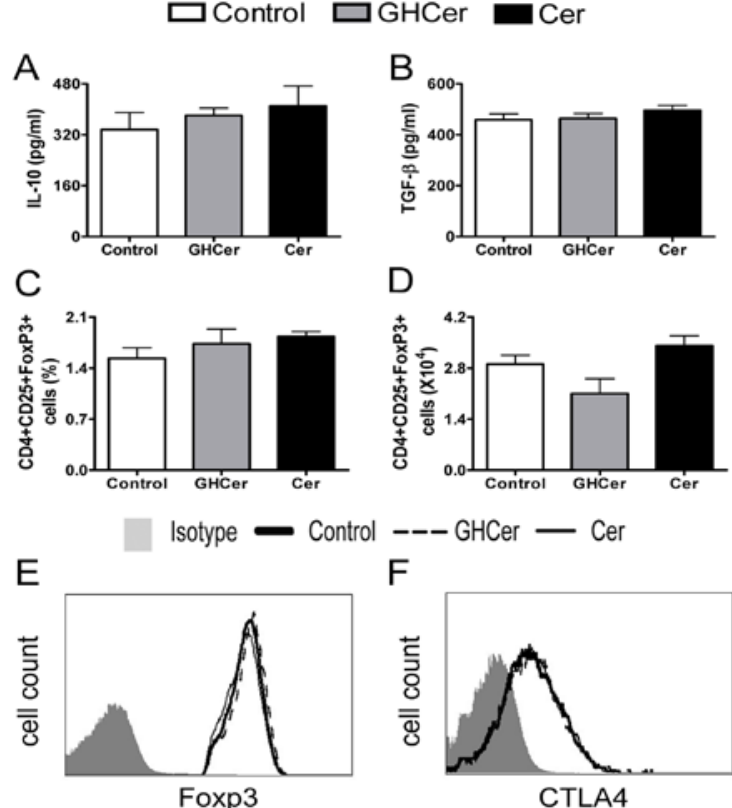

G
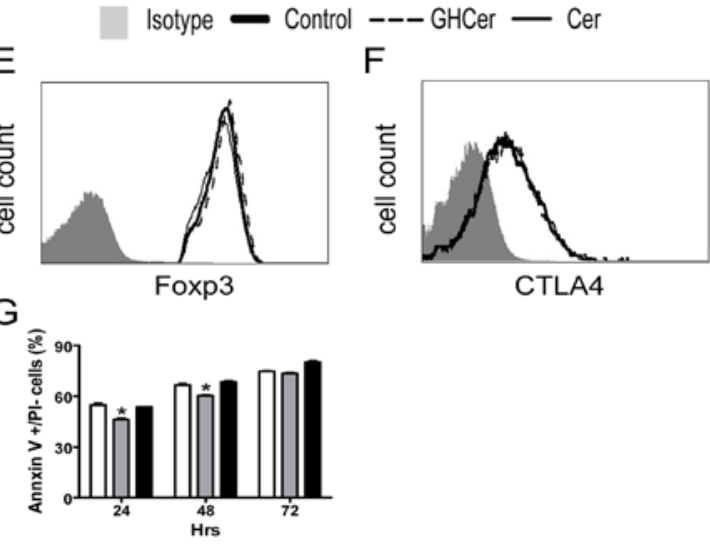

Figure 4: GHCer could neither expand Treg population nor induce apoptosis of splenocytes. Mouse splenocytes were incubated with GHCer, ceramide or medium control for $24 \mathrm{hrs}$, before activation by anti-mouse CD3/CD28 for 3 days. Supernatants were collected for determination of IL-10 (A) and TGF- $\beta$ (B) by ELISA assay. Cells were stained with antibodies against CD4, CD25, Foxp3 and CTLA4 at day2 and the percentage (C), absolute number (D), expression of Foxp3 (E) and CTLA4 (F) of Treg cells were determined. Splenocytes were harvested at the indicated time points for determination of apoptotic cells by staining with annexin $\mathrm{V}$ and $\mathrm{PI}$, followed by flow cytometric analysis. The percentage of apoptotic (annexin V+/PI-) cells was shown (G) and data are presented as mean \pm SD of triplicate. ${ }^{*}, p<0.05$, compared with control.

shown to modulate CD25 expression on T cells, we further analyzed the GHCer influence on Notch1 expression. As shown in Figures 5C and 5D, Notch1 expression in both mouse splenocytes and CD4+ T cells was inhibited by GHCer 2 days after stimulation by anti-CD3/ CD28 mAbs. Notch signaling was also essential for B cell differentiation into Ig-secreting plasma cells [27]. Hence we examined the influence of GHCer on Notch1 expression during B cell activation. Purified mouse CD19+ B cells were incubated with GHCer or ceramide before activation by LPS combined with IL- 4 and mouse CD40 ligand. On day2, Notch1 expression on CD19+ B cells was completely abolished by GHCer treatment when compared to the control or ceramide treated cells (Figure 5E). Similarly, GHcer also inhibited the upregulation of Notch1 expression on human CD4+ T cells during anti-CD3/CD28 mAbs (Figure $5 \mathrm{~F}$ ). These findings suggested that inhibition of immune cell proliferation and differentiation by GHCer might be attributed to the suppression of the Notch1 signaling.

\section{ID3 and Egr2/3 regulated E3 ubiquintin ligase may contribute} to Notch 1 suppression by GHCer

It was reported that Notch1 was negatively regulated at transcriptional level by ID3, the E2A antagonist [29] and at the protein 
level via ubiquintination by Itch [30]. Thus, we assessed the influence of GHCer on the expression of $i d 3$ and itch during $\mathrm{T}$ cell activation. Mouse splenic CD4+ T cells were incubated for 24 hrs with GHCer or ceramide, before activation by anti-mouse CD3/CD28 mAbs. RNA was collected at 2 and $12 \mathrm{hrs}$ to analyze the gene expression by real-time quantitative PCR. As shown in Figures $6 \mathrm{~A}$ and $6 \mathrm{~B}$, the expression of $i d 3$ and itch at 2 hrs was not affected by GHCer but raised to $2.1 \pm 0.2$ and $4.7 \pm 0.4$ folds higher, respectively, than in control cells at $12 \mathrm{hrs}$. As itch was regulated by Egr2/3 [35,36], we investigated the expression of egr $2 / 3$ after GHCer treatment. Treatment of CD $4+\mathrm{T}$ cells with GHCer increased the expression of egr 2 and egr 3 to $1.6 \pm 0.1$ and $1.2 \pm 0.03$ folds respectively, of control at $2 \mathrm{hrs}$ after activation which preceded itch upregulation (Figures 6C and 6D). Since overexpression of Egr2 and Egr3 also increased the expression of $c b l-b$, an E3 ubiquintin ligase [35], we determined $c b l-b$ expression in GHcer treated CD4+ T cells. As shown in Figure 6E, GHCer promoted the expression of $c b l-b$ to 2.1 \pm 0.3 folds of control at $12 \mathrm{hrs}$. In conclusion, these data suggested that GHCer could downregulate Notch1 expression through two pathways, one at transcriptional level by ID3, and the other at protein level through Egr2/3 controlled itch expression.

\section{Discussion}

GHCer is a glycosphingolipid of globo series, which is expressed on a variety of cancers $[2,3,37]$, but its function remains an enigma. In this study, we first demonstrated that human PBMC acquired globo $\mathrm{H}$ when co-cultured with globo $\mathrm{H}$ expressing cancer cells. GHCer could suppress proliferation and activation of human and murine immune cells which might contribute to the escape of cancer cells from immune surveillance. Previously, several cancer associated gangliosides were shown to induce immunosuppression [16]. To date, this is the first study to show that globoside can also inhibit immune activation. The length of the ceramide chain also played a role in the ganglioside induced immunosuppression. Gangliosides with shorter fatty acyl chain (C16:0, C18:0) was more immunosuppressive and easily shed

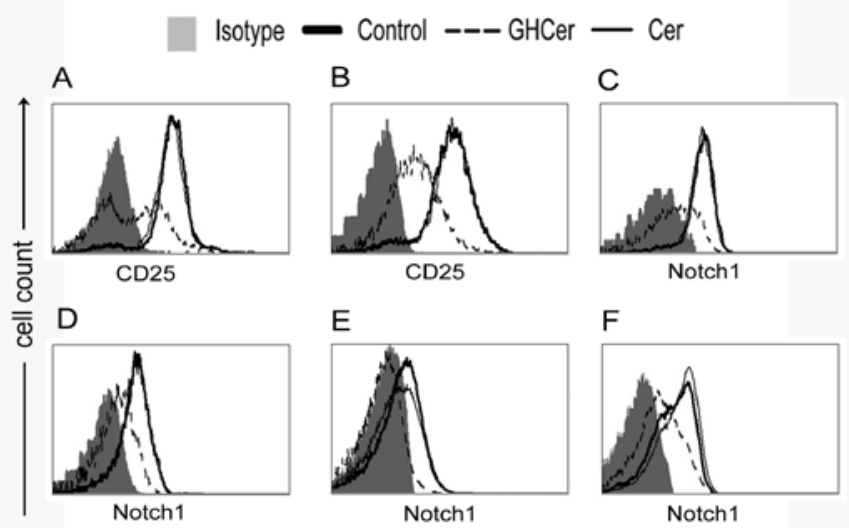

Figure 5: GHcer dampened the upregulation of CD25 and Notch1 during lymphocyte activation. Mouse splenocytes (A and $C$ ) and purified CD4+ T cells $(B$ and $D)$ were incubated with GHCer, ceramide or medium control for $24 \mathrm{hrs}$, and then activated by anti-mouse CD3 and CD28 mAb. Two days after activation, CD4 $+\mathrm{T}$ cells were gated to determine the expression of CD25 (A and $B$ ) and Notch1 (B and D) by flow cytometry. Purified mouse splenic CD19+ B cells were incubated with GHCer, ceramide or medium control for $24 \mathrm{hrs}$, and then activated by LPS combined with IL-4 and mouse CD40 ligand. Three days after activation, Notch1 expression was determined by flow cytometry (E). Human PBMC were incubate with GHCer or Cer for $24 \mathrm{hrs}$, and then activated by anti-human CD3 and CD28 mAb. Two days after activation, Notch1 expression on CD4+ T cells was determined by flow cytometry (F).

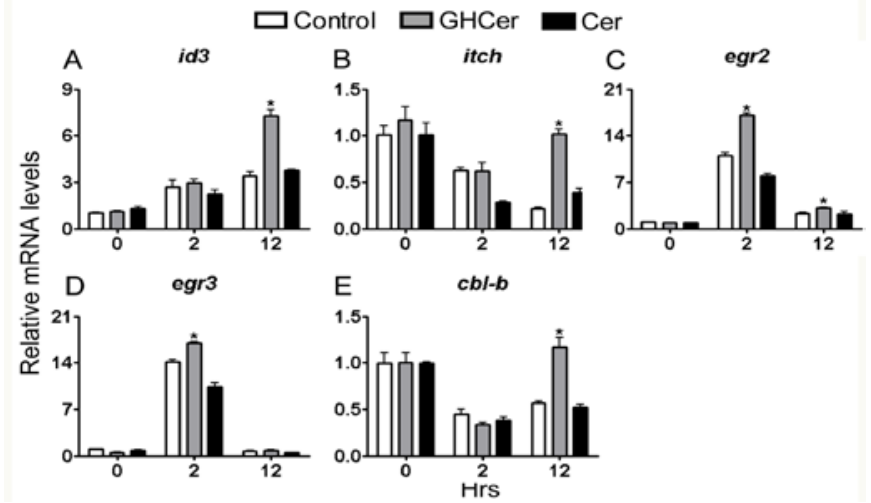

Figure 6: Effect of GHCer on the upregulation of id3 and Egr2/3-mediated increase of E3-ubiquintin ligases during $T$ cell activation. CD4+ T cells isolated from mouse spleen were incubated with $\mathrm{GHCer}$, ceramide or medium control for $24 \mathrm{hrs}$, and then activated by anti-CD3- and CD28 mAbs. At 2 and $12 \mathrm{hrs}$, total RNA was extracted for quantitation of id3 (A), itch (B), egr2 (C), egr3 (D), cbl-b (E) by real-time quantitative PCR. The mRNA levels were normalized to the level of $\beta$-actin and compared to resting cells. The data are presented as mean \pm SD of triplicate determinations. ${ }^{*}, p<0.05$, compared with medium control.

than those with a longer fatty acyl chain (C22:0/C24:1, C24:0) [38]. In MCF-7 cells, the most abundant fatty acyl chain linked to the globo $\mathrm{H}$ is C16:0 [39], and we showed that the synthetic GHCer with C16:0 fatty acyl chain indeed inhibited the activation of lymphocytes. Ceramide (C16:0) alone did not have any effect, suggesting that globo $\mathrm{H}$ residue was necessary for the immunosuppression.

Cancer cells can evade the host immune system by several mechanisms, such as shifting the microenvironment from Th1 to Th2 polarity [40], inducing the immunosuppressive cytokine IL-10 and TGF- $\beta$ [41], and regulatory T cells [42]. It had been shown that tumor derived gangliosides can shift theTh1/Th2 balance to promote the cancer progression. However, there were conflicting reports about the effects of various gangliosides on Th1 and Th 2 responses. For instance, Rayman et al. reported that GD1a derived from renal cell carcinoma inhibited the development of Th1 cell response by inducing the apoptosis of Th1 cytokine producing T cell [43]. Similarly, Crespo et al. indicated that GT1b, GD1a and GM1 shifted the immune response toward Th2 by inhibiting the IFN- $\gamma$ production and enhancing IL-4 dependent differentiation of $\mathrm{CD}^{+}{ }^{+} \mathrm{T}$ cells [21]. On the other hand, purified GT1b, GD1b and GQ1b had been reported to enhance the production of IL-2 and IFN- $\gamma$ but suppress IL-4 [44]. In addition, Kaushik et al. showed that gangliosides from some renal cell carcinoma patients suppressed both Th1 and Th2 cytokine responses [10]. So far, the effect of globo series glycosphingolipid on Th1 and Th2 cytokine has not been investigated. In this study, we demonstrated that acquisition of GHCer by immune cells decreased the production of IFN- $\gamma$, IL- 2 and IL-4 during T cell activation. Such suppressed Th1 and Th2 responses might create a microenvironment conducive to tumor growth. In short, the composition of carbohydrate moiety of glycosphingolipids on different tumors likely dictated the balance of Th1/Th2 activities, leading to tumor progression.

Regulatory $\mathrm{T}$ cells, formerly known as suppressor $\mathrm{T}$ cells, can potently inhibit the $\mathrm{T}$ cell proliferation. Our investigation showed that GHCer neither increased the regulatory $\mathrm{T}$ cell population, nor enhanced their expression of foxp3 and inhibitory molecular CTLA4. GHCer also failed to induce the suppressive cytokine IL-10 and TGF- $\beta$. These findings implied that GHCer-induced immunosuppression was 
Citation: Tsai YC, Huang JR, Cheng JY, Lin JJ, Hung JT, et al. (2013) A Prevalent Cancer Associated Glycan, Globo H Ceramide, Induces Immunosuppression by Reducing Notch1 Signaling. J Cancer Sci Ther 5: 264-270. doi:10.4172/1948-5956.1000215

not mediated by the Tregs. This is consistent with the previous finding that NGcGM3- induced immune suppression was mainly by reducing the functions of dendritic cells and CD4+CD25- T lymphocyte, rather than enhancing the regulatory $\mathrm{T}$ cell activity [45].

Although the cancer associated gangliosides-induced immune suppression had been reported by many studies, the molecular mechanisms were addressed by only a few studies which focused mainly on NF-kappa B. Irani et al. reported that brain derived gangliosides could inhibit the NF-kappa B activation in mitogen stimulated T cells [11]. Thornton et al. showed that gangliosides isolated from renal carcinoma suppressed the activation of NF-kappa B by degrading RelA and p50 proteins to impair nuclear localization of the p68/p50 NF-kappa B heterodimer in T cells [22]. Treatment of hepatocytes with GD3 blocked the activation of NF-kappa B and subsequent gene expression [46], implying that NF-kappa B played an important role in the gangliosides induced immunosuppression. However, the basis for the inhibition of NF-kappa B activation by gangliosides remains to be elucidated. NF-kappa B activity was reported to be modulated by several pathways, including Notch signaling. Shin et al. described that intracellular domain of Notch1 could directly interact with NF-kappa $\mathrm{B}$, leading to retention of NF-kappa B in the nucleus [47]. Cheng et al. also demonstrated that NF-kappa B activity was regulated by Notch1 via transcriptional control of NF-kappa B during B cell activation [48]. Notch signaling is crucial in determining $\mathrm{T}$ and $\mathrm{B}$ lymphoid lineage decision. Inhibition of Notch 1 cleavage in activated $\mathrm{T}$ cells resulted in decreased cell proliferation, and reduced production of IL- 2 and IFN- $\gamma$ [26]. Furthermore, the intracellular domain of Notch1 was shown to directly regulate IFN- $\gamma$ expression through complexes formed on the IFN- $\gamma$ promoter [47]. Our study provided the first evidence that cancer associated globo series glycosphingolipid, GHCer, could inhibit Notch1 expression during $\mathrm{T}$ and $\mathrm{B}$ cell activation. It will be of interest to investigate if gangliosides induced suppression of NF-kappa B is also mediated by reducing Notch 1 signaling. Such diminished Notch1 signaling may contribute to the GHCer-induced decreases in the cell proliferation, IL- 2 and IFN- $\gamma$ production and CD25 upregulation. Furthermore, we showed that treatment of T cells with GHCer boosted their expression of egr 2 and egr 3 , which in turns upregulated itch to degrade the Notch1 protein. The antagonist of E2A-mediated Notchl transcription, id 3 was also enhanced by GHCer. Henceforth, our findings indicated that GHCer inhibited T cell activation by enhancing the expression of $i d 3$ and itch, leading to diminished Notch 1 signaling. In addition to itch, Egr2/3 also regulated $c b l-b$ expression, which was found to be increased by GHCer treatment. In light of the report that ablation of $c b l-b$ in mice provided protection against engraftment of a variety of solid and hematopoietic tumors [49], the upregulation $c b l-b$ in T cells by GHCer might further dampen host immunity and favor tumor growth.

Egr2 and Egr3 are zinc-finger transcription factors of the early growth response gene family which negatively regulate $\mathrm{T}$ cell activation and induce $\mathrm{T}$ and $\mathrm{B}$ cell tolerance. Absence of full costimulation signal in TCR ligation would lead to induction of egr $2 / 3$ expression causing cell unresponsiveness $[35,50,51]$. The basis for our observed upregulation of egr $2 / 3$ by GHcer was not clear. It was possible that incorporation of GHCer in the immune cells might diminish costimulatory signaling or activate signaling molecules such as NFAT to induce egr $2 / 3$ expression [52]. Further investigation will be necessary to elucidate the underlying mechanisms.

Our findings provide a new insight that globo $\mathrm{H}$ released from cancer cells might alter the microenvironment in favor of tumor progression by suppressing the activation of immune cells. This was corroborated by our demonstration of globo $\mathrm{H}$ expression on tumorinfiltrating lymphocytes in a majority of globo $\mathrm{H}$ positive breast cancer tissues Thus, depletion of GHCer might be effective in overcoming immunosuppression with ensuing tumor regression. In view of the present study and our previous report that globo $\mathrm{H}$ was expressed on the breast cancer stem cells, globo $\mathrm{H}$ is an ideal target for cancer immunotherapy with globo $\mathrm{H}$ vaccine or anti-globo $\mathrm{H}$ monoclonal antibody. Our data further strengthened the scientific rationales for the ongoing phase II/III clinical trial of globo $\mathrm{H}$ vaccine in breast cancer.

\section{Acknowledgments}

We sincerely thank the cell imaging and flow cytometry core of the Division of Medical Biology, Genomics Research Center (Academia Sinica, Taipei, Taiwan) for providing excellent service assisted by Li-Wen Lo and Wen-Wen Chen. We are grateful to nurses Tzu-Yin Yeh and Hsiang-Min Kung for collecting clinical samples. We are indebted to Dr. Ragupathi for providing VK9 hybridoma and to OBI Pharma Inc.(Taipei, Taiwan) for supplying GHCer. This work was supported by Academia Sinica and National Science Council grants for A.L. Yu (NSC97-2323-B-001 and NSC 101-2325-B-001-022).

\section{References}

1. Kannagi R, Levery SB, Ishigami F, Hakomori S, Shevinsky LH, et al. (1983) New globoseries glycosphingolipids in human teratocarcinoma reactive with the monoclonal antibody directed to a developmentally regulated antigen stage-specific embryonic antigen 3. J Biol Chem 258: 8934-8942.

2. Zhang S, Zhang HS, Cordon-Cardo C, Reuter VE, Singhal AK, et al (1997) Selection of tumor antigens as targets for immune attack using immunohistochemistry: II. Blood group-related antigens. Int J Cancer 73: 5056.

3. Zhang S, Zhang HS, Reuter VE, Slovin SF, Scher HI, et al. (1998) Expression of potential target antigens for immunotherapy on primary and metastatic prostate cancers. Clin Cancer Res 4: 295-302.

4. Chang WW, Lee CH, Lee P, Lin J, Hsu CW, et al. (2008) Expression of Globo $\mathrm{H}$ and SSEA3 in breast cancer stem cells and the involvement of fucosyl transferases 1 and 2 in Globo H synthesis. Proc Natl Acad Sci U S A 105: 11667-11672.

5. Sabbatini PJ, Ragupathi G, Hood C, Aghajanian CA, Juretzka M, et al. (2007) Pilot study of a heptavalent vaccine-keyhole limpet hemocyanin conjugate plus QS21 in patients with epithelial ovarian, fallopian tube, or peritoneal cancer. Clin Cancer Res 13: 4170-4177.

6. Yu AL, Gilman AL, Ozkaynak MF, London WB, Kreissman SG, et al. (2010) AntiGD2 antibody with GM-CSF, interleukin-2, and isotretinoin for neuroblastoma N Engl J Med 363: 1324-1334.

7. Hakomori S (2001) Tumor-associated carbohydrate antigens defining tumo malignancy: basis for development of anti-cancer vaccines. Adv Exp Med Bio 491: 369-402.

8. Lang Z, Guerrera M, Li R, Ladisch S (2001) Ganglioside GD1a enhances VEGF-induced endothelial cell proliferation and migration. Biochem Biophys Res Commun 282: 1031-1037.

9. Chen HH, Fukumoto S, Furukawa K, Nakao A, Akiyama S, et al. (2003) Suppression of lung metastasis of mouse Lewis lung cancer P29 with transfection of the ganglioside GM2/GD2 synthase gene. Int J Cancer 103 169-176.

10. Biswas K, Richmond A, Rayman P, Biswas S, Thornton M, et al. (2006) GM2 expression in renal cell carcinoma: potential role in tumor-induced T-cell dysfunction. Cancer Res 66: 6816-6825.

11. Irani DN, Lin KI, Griffin DE (1996) Brain-derived gangliosides regulate the cytokine production and proliferation of activated T cells. J Immunol 157: 43334340 .

12. Péguet-Navarro J, Sportouch M, Popa I, Berthier O, Schmitt D, et al. (2003) Gangliosides from human melanoma tumors impair dendritic cell differentiation from monocytes and induce their apoptosis. J Immunol 170: 3488-3494.

13. Biswas S, Biswas K, Richmond A, Ko J, Ghosh S, et al. (2009) Elevated levels of select gangliosides in T cells from renal cell carcinoma patients is associated with T cell dysfunction. J Immunol 183: 5050-5058.

14. Chu JW, Sharom FJ (1993) Gangliosides inhibit T-lymphocyte proliferation 
Citation: Tsai YC, Huang JR, Cheng JY, Lin JJ, Hung JT, et al. (2013) A Prevalent Cancer Associated Glycan, Globo H Ceramide, Induces Immunosuppression by Reducing Notch1 Signaling. J Cancer Sci Ther 5: 264-270. doi:10.4172/1948-5956.1000215

by preventing the interaction of interleukin-2 with its cell surface receptors. Immunology 79: 10-17.

15. Morioka N, Furue M, Tsuchida T, Ishibashi Y (1991) Gangliosides inhibit the proliferation of human T cells stimulated with interleukin-4 or interleukin-2. J Dermatol 18: 447-453.

16. Ladisch S, Becker H, Ulsh L (1992) Immunosuppression by human gangliosides: I. Relationship of carbohydrate structure to the inhibition of $\mathrm{T}$ cell responses. Biochim Biophys Acta 1125: 180-188.

17. Uzzo RG, Rayman P, Kolenko V, Clark PE, Cathcart MK, et al. (1999) Rena cell carcinoma-derived gangliosides suppress nuclear factor-kappaB activation in T cells. J Clin Invest 104: 769-776.

18. Wölfl M, Batten WY, Posovszky C, Bernhard H, Berthold F (2002) Gangliosides inhibit the development from monocytes to dendritic cells. Clin Exp Immunol 130: 441-448.

19. Lu P, Sharom FJ (1996) Immunosuppression by YAC-1 lymphoma: role of shed gangliosides. Cell Immunol 173: 22-32.

20. Das T, Sa G, Hilston C, Kudo D, Rayman P, et al. (2008) GM1 and tumor necrosis factor-alpha, overexpressed in renal cell carcinoma, synergize to induce T-cell apoptosis. Cancer Res 68: 2014-2023.

21. Crespo FA, Sun X, Cripps JG, Fernandez-Botran R (2006) The immunoregulatory effects of gangliosides involve immune deviation favoring type-2 T cell responses. J Leukoc Biol 79: 586-595.

22. Thornton MV, Kudo D, Rayman P, Horton C, Molto L, et al. (2004) Degradation of NF-kappa B in T cells by gangliosides expressed on renal cell carcinomas. J Immunol 172: 3480-3490.

23. Osipo C, Golde TE, Osborne BA, Miele LA (2008) Off the beaten pathway: the complex cross talk between Notch and NF-kappaB. Lab Invest 88: 11-17.

24. Artavanis-Tsakonas S, Rand MD, Lake RJ (1999) Notch signaling: cell fate control and signal integration in development. Science 284: 770-776.

25. Allman D, Punt JA, Izon DJ, Aster JC, Pear WS (2002) An invitation to T and more: notch signaling in lymphopoiesis. Cell 109 Suppl: S1-11.

26. Palaga T, Miele L, Golde TE, Osborne BA (2003) TCR-mediated Notch signaling regulates proliferation and IFN-gamma production in peripheral $\mathrm{T}$ cells. J Immunol 171: 3019-3024.

27. Santos MA, Sarmento LM, Rebelo M, Doce AA, Maillard I, et al. (2007) Notch1 engagement by Delta-like-1 promotes differentiation of B lymphocytes to antibody-secreting cells. Proc Natl Acad Sci U S A 104: 15454-15459.

28. Kopan R, llagan MX (2009) The canonical Notch signaling pathway: unfolding the activation mechanism. Cell 137: 216-233.

29. Yashiro-Ohtani Y, He Y, Ohtani T, Jones ME, Shestova O, et al. (2009) PreTCR signaling inactivates Notch1 transcription by antagonizing E2A. Genes Dev 23: 1665-1676.

30. Qiu L, Joazeiro C, Fang N, Wang HY, Elly C, et al. (2000) Recognition and ubiquitination of Notch by Itch, a hect-type E3 ubiquitin ligase. J Biol Chem 275: 35734-35737

31. Adler SH, Chiffoleau E, Xu L, Dalton NM, Burg JM, et al. (2003) Notch signaling augments $\mathrm{T}$ cell responsiveness by enhancing CD25 expression. J Immunol 171: 2896-2903

32. Chang F, Li R, Ladisch S (1997) Shedding of gangliosides by human medulloblastoma cells. Exp Cell Res 234: 341-346.

33. Santin AD, Ravindranath $\mathrm{MH}$, Bellone S, Muthugounder S, Palmieri M, et al. (2004) Increased levels of gangliosides in the plasma and ascitic fluid of patients with advanced ovarian cancer. BJOG 111: 613-618.

34. McKallip R, Li R, Ladisch S (1999) Tumor gangliosides inhibit the tumor-specific immune response. J Immunol 163: 3718-3726.

35. Safford M, Collins S, Lutz MA, Allen A, Huang CT, et al. (2005) Egr-2 and Egr-3 are negative regulators of $T$ cell activation. Nat Immunol 6: 472-480.

36. Chang X, Chen L, Wen J, Godfrey VL, Qiao G, et al. (2006) Foxp3 controls autoreactive $T$ cell activation through transcriptional regulation of early growth response genes and E3 ubiquitin ligase genes, independently of thymic selection. Clin Immunol 121: 274-285.

37. Menard S, Tagliabue E, Canevari S, Fossati G, Colnaghi MI (1983) Generation of monoclonal antibodies reacting with normal and cancer cells of human breast. Cancer Res 43: 1295-1300.

38. Ladisch S, Li R, Olson E (1994) Ceramide structure predicts tumor ganglioside immunosuppressive activity. Proc Natl Acad Sci U S A 91: 1974-1978.

39. Bremer EG, Levery SB, Sonnino S, Ghidoni R, Canevari S, et al. (1984) Characterization of a glycosphingolipid antigen defined by the monoclonal antibody $\mathrm{MBr} 1$ expressed in normal and neoplastic epithelial cells of human mammary gland. J Biol Chem 259: 14773-14777.

40. Tatsumi T, Kierstead LS, Ranieri E, Gesualdo L, Schena FP, et al. (2002) Disease-associated bias in T helper type 1 (Th1)/Th2 CD4(+) T cell responses against MAGE-6 in HLA-DRB10401(+) patients with renal cell carcinoma or melanoma. J Exp Med 196: 619-628.

41. Toutirais $O$, Chartier $P$, Dubois $D$, Bouet $F$, Lévêque J, et al. (2003) Constitutive expression of TGF-bêta1, interleukin- 6 and interleukin- 8 by tumor cells as a major component of immune escape in human ovarian carcinoma. Eur Cytokine Netw 14: 246-255.

42. Zou W (2006) Regulatory T cells, tumour immunity and immunotherapy. Nat Rev Immunol 6: 295-307.

43. Rayman P, Wesa AK, Richmond AL, Das T, Biswas K, et al. (2004) Effect of renal cell carcinomas on the development of type $1 \mathrm{~T}$-cell responses. Clin Cancer Res 10: 6360S-6S

44. Kanda N, Watanabe S (2001) Gangliosides GD1b, GT1b, and GQ1b enhance IL-2 and IFN-gamma production and suppress IL-4 and IL-5 production in phytohemagglutinin-stimulated human T cells. J Immunol 166: 72-80.

45. de León J, Fernández A, Clavell M, Labrada M, Bebelagua Y, et al. (2008) Differential influence of the tumour-specific non-human sialic acid containing GM3 ganglioside on CD4+CD25- effector and naturally occurring CD4+CD25+ regulatory T cells function. Int Immunol 20: 591-600.

46. Colell A, García-Ruiz C, Roman J, Ballesta A, Fernández-Checa JC (2001) Ganglioside GD3 enhances apoptosis by suppressing the nuclear factor-kappa B-dependent survival pathway. FASEB J 15: 1068-1070.

47. Shin HM, Minter LM, Cho OH, Gottipati S, Fauq AH, et al. (2006) Notch1 augments NF-kappaB activity by facilitating its nuclear retention. EMBO J 25 129-138.

48. Cheng P, Zlobin A, Volgina V, Gottipati S, Osborne B, et al. (2001) Notch-1 regulates NF-kappaB activity in hemopoietic progenitor cells. J Immunol 167 4458-4467.

49. Chiang JY, Jang IK, Hodes R, Gu H (2007) Ablation of Cbl-b provides protection against transplanted and spontaneous tumors. J Clin Invest 117: 1029-1036.

50. Harris JE, Bishop KD, Phillips NE, Mordes JP, Greiner DL, et al. (2004) Early growth response gene-2, a zinc-finger transcription factor, is required for ful induction of clonal anergy in CD4+ T cells. J Immunol 173: 7331-7338.

51. Schwartz RH (1996) Models of T cell anergy: is there a common molecular mechanism? J Exp Med 184: 1-8.

52. Rengarajan J, Mittelstadt PR, Mages HW, Gerth AJ, Kroczek RA, et al. (2000) Sequential involvement of NFAT and Egr transcription factors in FasL regulation. Immunity 12: 293-300. 